\title{
Global nonexistence of solutions for nonlinear coupled viscoelastic wave equations with damping and source terms
}

Jianghao Hao*, Shasha Niu and Huihui Meng

\section{"Correspondence:} hjhao@sxu.edu.cn School of Mathematical Sciences, Shanxi University, Taiyuan, Shanxi 030006, P.R. China

\begin{abstract}
In this paper, we are concerned with a nonlinear coupled viscoelastic wave equations with initial-boundary value conditions and nonlinear damping and source terms. Under suitable assumptions on relaxation functions, damping terms, and source terms, by using the energy method we proved a global nonexistence result for certain solutions with negative initial energy.
\end{abstract}

Keywords: coupled viscoelastic wave equations; relaxation functions; damping terms; source terms; global nonexistence

\section{Introduction}

In this paper, we study the following initial-boundary value coupled viscoelastic problem:

$$
\left\{\begin{array}{l}
\left|u_{t}\right|^{j} u_{t t}-\Delta u_{t t}-\operatorname{div}\left(|\nabla u|^{\alpha-2} \nabla u\right)-\Delta u+\int_{0}^{t} g(t-s) \Delta u d s \\
\quad+\left|u_{t}\right|^{m-1} u_{t}=f_{1}(u, v), \quad(x, t) \in \Omega \times(0, T), \\
\left|v_{t}\right|^{j} v_{t t}-\Delta v_{t t}-\operatorname{div}\left(|\nabla v|^{\beta-2} \nabla v\right)-\Delta v+\int_{0}^{t} h(t-s) \Delta v d s \\
\quad+\left|v_{t}\right|^{r-1} v_{t}=f_{2}(u, v), \quad(x, t) \in \Omega \times(0, T), \\
u(x, t)=v(x, t)=0, \quad(x, t) \in \partial \Omega \times(0, T), \\
u(x, 0)=u_{0}(x), \quad u_{t}(x, 0)=u_{1}(x), \quad x \in \Omega, \\
v(x, 0)=v_{0}(x), \quad v_{t}(x, 0)=v_{1}(x), \quad x \in \Omega,
\end{array}\right.
$$

where $\Omega$ is a bounded domain in $R^{n}$ with smooth boundary $\partial \Omega$, the constants $j>0, \alpha \geq 2$, $\beta \geq 2, m \geq 1, r \geq 1$, the nonlinear functions $f_{1}(u, v)$ and $f_{2}(u, v)$ are

$$
\begin{aligned}
& f_{1}(u, v)=a|u+v|^{2(p+1)}(u+v)+b|u|^{p} u|v|^{p+2}, \\
& f_{2}(u, v)=a|u+v|^{2(p+1)}(u+v)+b|u|^{p+2}|v|^{p} v,
\end{aligned}
$$

in which the constants $a>0, b>0$, and $p$ satisfies

$$
\begin{cases}p>-1, & n=1,2, \\ -1<p \leq \frac{4-n}{n-2}, & n \geq 3 .\end{cases}
$$

The pioneering work of Dafermos [1] studied a one-dimensional viscoelastic problem, established some existence and asymptotic stability results for smooth monotone decreas-

\section{并 Springer}

(c) 2014 Hao et al.; licensee Springer. This is an Open Access article distributed under the terms of the Creative Commons Attribution License (http://creativecommons.org/licenses/by/4.0), which permits unrestricted use, distribution, and reproduction in any medium, provided the original work is properly credited. 
ing relaxation functions. Hrusa [2] established several global existence and asymptotic stability results for a semilinear hyperbolic Volterra equation which allows the initial data to be large. Muñoz Rivera [3] considered equations for linear isotropic viscoelastic solids of integral type, and established exponential decay and polynomial decay in a bounded domain and in the whole space, respectively. After that, many results of existence and longterm behavior have been established. Messaoudi [4] considered a nonlinear viscoelastic wave equation with source and damping terms of the form

$$
u_{t t}-\Delta u+\int_{0}^{t} g(t-s) \Delta u(s) d s+u_{t}\left|u_{t}\right|^{m-1}=u|u|^{p-1} .
$$

He established blow-up result for solutions with negative initial energy and $m<p$, and gave a global existence result for arbitrary initial (in the appropriate space) if $m \geq p$. This work was later improved by Messaoudi [5].

Cavalcanti and Oquendo [6] discussed the equation

$$
u_{t t}-k_{0} \Delta u+\int_{0}^{t} \operatorname{div}[a(x) g(t-\tau) \nabla u(\tau)] d \tau+b(x) h\left(u_{t}\right)+f(u)=0,
$$

under some conditions on the relaxation function $g$ and $a(x)+b(x) \geq \delta>0$. They proved an exponential stability result when the relaxation function $g$ is decaying exponentially and the function $h$ is linear and a polynomial stability when $g$ is decaying polynomially and $h$ is nonlinear.

Cavalcanti et al. [7] discussed the wave equation

$$
u_{t t}-\Delta u+\int_{0}^{t} g(t-\tau) \operatorname{div}[a(x) \nabla u(\tau)] d \tau+b(x) f\left(u_{t}\right)=0
$$

on a compact Riemannian manifold $(M, \mathbf{g})$ subject to a combination of locally distributed viscoelastic and frictional dissipations. It is shown that the solutions decay according to the law dictated by the decay rates corresponding to the slowest damping.

Muñoz Rivera and Naso [8] studied a viscoelastic systems with nondissipative kernels and showed that if the kernel function decays exponentially to zero, then the solution decays exponentially to zero. On the other hand, if the kernel function decays polynomially as $t^{-p}$, then the corresponding solution also decays polynomially to zero with the same rate of decay.

Wang and Wang [9] studied a one-dimensional wave equation with viscoelastic damping under the Dirichlet boundary condition, where the kernel was taken for the finite sum of exponential polynomials. Using the asymptotic analysis technique, the authors proved an exponential stability result. Zhao and Wang [10] considered a coupled system of an ODE and a wave equation with Kelvin-Voigt damping, where the velocity of the wave equation entered the ODE and the output was fed into the boundary of the wave equation. They presented the asymptotic expressions of eigenvalues and eigenfunctions and established the exponential stability result.

Cavalcanti et al. [11] discussed a quasilinear initial-boundary value problem:

$$
\left|u_{t}\right|^{\rho} u_{t t}-\Delta u-\Delta u_{t t}+\int_{0}^{t} g(t-s) \Delta u(\tau) d \tau-\gamma \Delta u_{t}=b u|u|^{p-2},
$$


with Dirichlet boundary condition, where $\rho>0, \gamma \geq 0, p \geq 2, b=0$. An exponential decay result for $\gamma>0$ and $b=0$ has been obtained. For $\gamma=0$ and $b>0$, Messaoudi and Tatar $[12,13]$ showed that there exists an appropriate set, called stable set, such that if the initial data are in stable set, the solution continues to live there forever, and the solution goes to zero with an exponential or polynomial rate depending on the decay rate of relaxation function.

Pata [14] studied the abstract integrodifferential equations

$$
u_{t t}+\alpha A u+\beta u_{t}-\int_{0}^{t} g(\tau) A u(t-\tau) d \tau=0
$$

for $\alpha>0, \beta \geq 0, g$ is a positive summable kernel, $H$ be a real Hilbert space and the operator $A: D(A) \rightarrow H$ be a self-adjoint linear positive definite operator with domain $D(A) \subset H$ such that the embedding is dense and compact. He introduced some new concepts such as the flatness of a kernel and gave the asymptotic result.

Lasiecka et al. [15] discussed (1.6) with $\alpha=1, \beta=0$ with memory kernel satisfying the inequality $g^{\prime}+H(g) \leq 0, s \geq 0$ where $H(s)$ is a given continuous positive increasing and convex function such that $H(0)=0$. They developed an intrinsic method for determining decay rates of the energy given in terms of the function $H(s)$.

Han and Wang [16] studied the following initial-boundary value problem for a coupled system of nonlinear viscoelastic equations:

$$
\left\{\begin{array}{l}
u_{t t}-\Delta u+\int_{0}^{t} g_{1}(t-\tau) \Delta u(\tau) d \tau+\left|u_{t}\right|^{m-1} u_{t}=f_{1}(u, v), \quad(x, t) \in \Omega \times(0, T), \\
v_{t t}-\Delta v+\int_{0}^{t} g_{2}(t-\tau) \Delta v(\tau) d \tau+\left|v_{t}\right|^{m-1} v_{t}=f_{2}(u, v), \quad(x, t) \in \Omega \times(0, T), \\
u=v=0, \quad(x, t) \in \partial \Omega \times(0, T), \\
u(x, 0)=u_{0}(x), \quad u_{t}(x, 0)=u_{1}(x), \quad x \in \Omega, \\
v(x, 0)=v_{0}(x), \quad v_{t}(x, 0)=v_{1}(x), \quad x \in \Omega .
\end{array}\right.
$$

Local existence, global existence, uniqueness, and blow-up in finite time were obtained when $f_{1}, f_{2}, g_{1}, g_{2}$, and the initial values satisfy some conditions.

Messaoudi and Said-Houari [17] dealt with the problem (1.7) and proved a global nonexistence of solutions for a large class of initial data for which the initial energy takes positive values. Also, Said-Houari et al. [18] discussed (1.7) and proved a general decay result.

Liu [19] considered the following initial-boundary value problem for a coupled system of quasilinear viscoelastic equations:

$$
\left\{\begin{array}{l}
\left|u_{t}\right|^{\rho} u_{t t}-\Delta u-c_{1} \Delta u_{t t}+\int_{0}^{t} g_{1}(t-\tau) \Delta u(\tau) d \tau+f(u, v)=0, \quad(x, t) \in \Omega \times(0, \infty), \\
\left|v_{t}\right|^{\rho} v_{t t}-\Delta v-c_{2} \Delta v_{t t}+\int_{0}^{t} g_{2}(t-\tau) \Delta v(\tau) d \tau+k(u, v)=0, \quad(x, t) \in \Omega \times(0, \infty), \\
u=v=0, \quad(x, t) \in \partial \Omega \times(0, \infty), \\
u(x, 0)=u_{0}(x), \quad u_{t}(x, 0)=u_{1}(x), \quad x \in \Omega, \\
v(x, 0)=v_{0}(x), \quad v_{t}(x, 0)=v_{1}(x), \quad x \in \Omega .
\end{array}\right.
$$

The author used perturbed energy method to show that dissipations given by the viscoelastic terms are strong enough to ensure the decay of the corresponding energy function.

Our purpose in this paper is to give the global nonexistence of solutions for coupled viscoelastic equations with damping and source terms by using the energy method. 
The present work is organized as follows. In Section 2, we give some notations and material needed for this work. Section 3 contains the main result and the proof of the global nonexistence result.

\section{Preliminary}

In this section, we give some notations and material needed for the proof of our result.

We shall write $\|\cdot\|_{p}$ to denote the usual $L^{p}(\Omega)$ norm $\|\cdot\|_{L^{p}(\Omega)}$ and $\|\cdot\|$ to denote the usual $L^{2}(\Omega)$ norm $\|\cdot\|_{L^{2}(\Omega)}$.

First, we make the following assumptions on the $C^{1}$-nonnegative and nonincreasing relaxation functions $g$ and $h$ :

$$
1-\int_{0}^{\infty} g(s) d s=l>0, \quad 1-\int_{0}^{\infty} h(s) d s=k>0 .
$$

One can easily verify for $(\xi, \eta) \in R^{2}$,

$$
\begin{aligned}
& \xi f_{1}(\xi, \eta)+\eta f_{2}(\xi, \eta)=2(p+2) F(\xi, \eta), \\
& F(\xi, \eta)=\frac{1}{2(p+2)}\left[a|\xi+\eta|^{2(p+2)}(\xi+\eta)+2 b|\xi \eta|^{p+2}\right]
\end{aligned}
$$

and

$$
f_{1}(\xi, \eta)=\frac{\partial F(\xi, \eta)}{\partial \xi}, \quad f_{2}(\xi, \eta)=\frac{\partial F(\xi, \eta)}{\partial \eta} .
$$

Define

$$
(\phi \circ \psi)(t):=\int_{0}^{t} \phi(t-\tau) \int_{\Omega}|\psi(t)-\psi(\tau)|^{2} d x d \tau
$$

and

$$
\begin{aligned}
E(t):= & \frac{1}{j+2}\left(\left\|u_{t}\right\|_{j+2}^{j+2}+\left\|v_{t}\right\|_{j+2}^{j+2}\right)+\frac{1}{2}\left(\left\|\nabla u_{t}\right\|^{2}+\left\|\nabla v_{t}\right\|^{2}\right) \\
& +\frac{1}{2}\left(1-\int_{0}^{t} g(s) d s\right)\|\nabla u\|^{2}+\frac{1}{2}\left(1-\int_{0}^{t} h(s) d s\right)\|\nabla v\|^{2} \\
& -\int_{\Omega} F(u, v) d x+\frac{1}{2}(g \circ \nabla u+h \circ \nabla v)+\frac{1}{\alpha}\|\nabla u\|_{\alpha}^{\alpha}+\frac{1}{\beta}\|\nabla v\|_{\beta}^{\beta} .
\end{aligned}
$$

\section{Global nonexistence result}

In this section, we give the global nonexistence result and its proof.

Theorem 3.1 Assume that

$$
\max \{j+1, m+1, r+1\}<2(p+2), \quad E(0)<0,
$$

and there exists constant $\gamma$ such that

$\max \{\alpha, \beta\}<\gamma<2(p+2)$ 
and

$$
\min \{l, k\}>\frac{1 /(2 \gamma)}{(\gamma / 2)-1+1 /(2 \gamma)} .
$$

Then any solution of (1.1) cannot exist for all time.

Proof By multiplying the first and second equation of (1.1) by $u_{t}$ and $v_{t}$, respectively, integrating over $\Omega$ and summing them up, we obtain

$$
\begin{aligned}
\frac{d}{d t} E(t)= & -\left(\left\|u_{t}\right\|_{m+1}^{m+1}+\left\|v_{t}\right\|_{r+1}^{r+1}\right)+\frac{1}{2}\left(g^{\prime} \circ \nabla u+h^{\prime} \circ \nabla v\right) \\
& -\frac{1}{2}\left(g(t)\|\nabla u\|^{2}+h(t)\|\nabla v\|^{2}\right) .
\end{aligned}
$$

Define

$$
H(t):=-E(t)
$$

then

$$
0<H(0) \leq H(t) \leq \int_{\Omega} F(u, v) d x \leq \frac{C_{1}}{2(p+2)}\left(\|u\|_{2(p+2)}^{2(p+2)}+\|v\|_{2(p+2)}^{2(p+2)}\right) .
$$

Throughout, $C$ and $C_{i}$ represent generic positive constants.

Define

$$
\Theta(t):=H^{1-\delta}(t)+\frac{\varepsilon}{j+1} \int_{\Omega}\left(\left|u_{t}\right|^{j} u_{t} u+\left|v_{t}\right|^{j} v_{t} v\right) d x-\varepsilon \int_{\Omega}\left(\Delta u u_{t}+\Delta v v_{t}\right) d x
$$

where $\varepsilon$ is a positive constant to be determined, and

$$
\begin{aligned}
0 & <\delta \\
& \leq \min \left\{\frac{2(p+2)-(j+2)}{2(j+2)(p+2)}, \frac{2(p+2)-(m+1)}{2 m(p+2)}, \frac{2(p+2)-(r+1)}{2 r(p+2)}, \frac{\alpha-2}{2 \alpha}, \frac{\beta-2}{2 \beta}\right\} .
\end{aligned}
$$

By differentiation, we obtain

$$
\begin{aligned}
\Theta^{\prime}(t)= & (1-\delta) H^{-\delta}(t) H^{\prime}(t)+\varepsilon \int_{\Omega}\left[\left(\left|u_{t}\right|^{j} u_{t t} u+\left|v_{t}\right|^{j} v_{t t} v\right)+\frac{1}{j+1}\left(\left|u_{t}\right|^{j+2}+\left|v_{t}\right|^{j+2}\right)\right] d x \\
& +\varepsilon\left(\left\|\nabla u_{t}\right\|^{2}+\left\|\nabla v_{t}\right\|^{2}\right)-\varepsilon \int_{\Omega}\left(u \Delta u_{t t}+v \Delta v_{t t}\right) d x \\
= & (1-\delta) H^{-\delta}(t) H^{\prime}(t)+\frac{\varepsilon}{j+1}\left(\left\|u_{t}\right\|_{j+2}^{j+2}+\left\|v_{t}\right\|_{j+2}^{j+2}\right) \\
& -\varepsilon \int_{\Omega}\left(\left|u_{t}\right|^{m-1} u_{t} u+\left|v_{t}\right|^{r-1} v_{t} v\right) d x+\varepsilon\left(\left\|\nabla u_{t}\right\|^{2}+\left\|\nabla v_{t}\right\|^{2}\right) \\
& -\varepsilon\left(\|\nabla u\|^{2}+\|\nabla v\|^{2}\right)-\varepsilon\left(\|\nabla u\|_{\alpha}^{\alpha}+\|\nabla v\|_{\beta}^{\beta}\right)+2 \varepsilon(p+2) \int_{\Omega} F(u, v) d x \\
& +\varepsilon\left(\int_{0}^{t} g(s) d s\right)\|\nabla u\|^{2}+\varepsilon\left(\int_{0}^{t} h(s) d s\right)\|\nabla v\|^{2}
\end{aligned}
$$




$$
\begin{aligned}
& +\varepsilon \int_{0}^{t} g(t-s) \int_{\Omega} \nabla u(t)[\nabla u(s)-\nabla u(t)] d x d s \\
& +\varepsilon \int_{0}^{t} h(t-s) \int_{\Omega} \nabla v(t)[\nabla v(s)-\nabla v(t)] d x d s .
\end{aligned}
$$

Using Hölder's inequality and Young's inequality, we get

$$
\begin{gathered}
\int_{0}^{t} g(t-s) \int_{\Omega} \nabla u(t)[\nabla u(s)-\nabla u(t)] d x d s \\
\leq \int_{0}^{t} g(t-s)\|\nabla u(t)\|\|\nabla u(s)-\nabla u(t)\| d s \\
\leq \lambda(g \circ \nabla u)+\frac{1}{4 \lambda}\left(\int_{0}^{t} g(s) d s\right)\|\nabla u\|^{2}, \\
\int_{0}^{t} h(t-s) \int_{\Omega} \nabla v(t)[\nabla v(s)-\nabla v(t)] d x d s \\
\leq \int_{0}^{t} h(t-s)\|\nabla v(t)\|\|\nabla v(s)-\nabla v(t)\| d s \\
\leq \lambda(h \circ \nabla v)+\frac{1}{4 \lambda}\left(\int_{0}^{t} h(s) d s\right)\|\nabla v\|^{2},
\end{gathered}
$$

for $0<\lambda<\frac{\gamma}{2}$, thus

$$
\begin{aligned}
\Theta^{\prime}(t) \geq & (1-\delta) H^{-\delta}(t) H^{\prime}(t)+\varepsilon\left(\frac{1}{j+1}+\frac{\gamma}{j+2}\right)\left(\left\|u_{t}\right\|_{j+2}^{j+2}+\left\|v_{t}\right\|_{j+2}^{j+2}\right) \\
& -\varepsilon \int_{\Omega}\left(\left|u_{t}\right|^{m-1} u_{t} u+\left|v_{t}\right|^{r-1} v_{t} v\right) d x+\varepsilon\left(1+\frac{\gamma}{2}\right)\left(\left\|\nabla u_{t}\right\|^{2}+\left\|\nabla v_{t}\right\|^{2}\right) \\
& +\gamma \varepsilon H(t)+\varepsilon(2(p+2)-\gamma) \int_{\Omega} F(u, v) d x \\
& +\varepsilon\left[\left(\frac{\gamma}{2}-1\right)-\left(\frac{\gamma}{2}-1+\frac{1}{4 \lambda}\right) \int_{0}^{\infty} g(s) d s\right]\|\nabla u\|^{2} \\
& +\varepsilon\left[\left(\frac{\gamma}{2}-1\right)-\left(\frac{\gamma}{2}-1+\frac{1}{4 \lambda}\right) \int_{0}^{\infty} h(s) d s\right]\|\nabla v\|^{2} \\
& +\varepsilon\left(\frac{\gamma}{2}-\lambda\right)(g \circ \nabla u+h \circ \nabla v)+\varepsilon\left(\frac{\gamma}{\alpha}-1\right)\|\nabla u\|_{\alpha}^{\alpha}+\varepsilon\left(\frac{\gamma}{\beta}-1\right)\|\nabla v\|_{\beta}^{\beta} .
\end{aligned}
$$

By Young's inequality, we get

$$
\begin{aligned}
\int_{\Omega}\left|u_{t}\right|^{m-1} u_{t} u d x & \leq \frac{\delta_{1}^{m+1}}{m+1}\|u\|_{m+1}^{m+1}+\frac{m \delta_{1}^{-\frac{m+1}{m}}}{m+1}\left\|u_{t}\right\|_{m+1}^{m+1} \\
& \leq \frac{\delta_{1}^{m+1}}{m+1}\|u\|_{m+1}^{m+1}+\frac{m \delta_{1}^{-\frac{m+1}{m}}}{m+1} H^{\prime}(t)
\end{aligned}
$$

and

$$
\int_{\Omega}\left|v_{t}\right|^{r-1} v_{t} v d x \leq \frac{\delta_{2}^{r+1}}{r+1}\|v\|_{r+1}^{r+1}+\frac{r \delta_{2}^{-\frac{r+1}{r}}}{r+1}\left\|v_{t}\right\|_{r+1}^{r+1} \leq \frac{\delta_{2}^{r+1}}{r+1}\|v\|_{r+1}^{r+1}+\frac{r \delta_{2}^{-\frac{r+1}{r}}}{r+1} H^{\prime}(t) .
$$


Set

$$
\delta_{1}^{-\frac{m+1}{m}}=M_{1} H^{-\delta}, \quad \delta_{2}^{-\frac{r+1}{r}}=M_{2} H^{-\delta}
$$

in which $M_{1}$ and $M_{2}$ will be specified later, and $\gamma$ obeys

$$
\min \left\{\frac{\gamma}{\alpha}-1, \frac{\gamma}{\beta}-1\right\}>0
$$

and

$$
1+\frac{\gamma}{2}>0
$$

Then due to (3.2), we get

$$
\delta_{1}^{m+1}=M_{1}^{-m} H^{m \delta} \leq \frac{M_{1}^{-m} C_{1}^{m \delta}}{(2(p+2))^{m \delta}}\left(\|u\|_{2(p+2)}^{2(p+2)}+\|v\|_{2(p+2)}^{2(p+2)}\right)^{m \delta}
$$

and

$$
\delta_{2}^{r+1}=M_{2}^{-r} H^{r \delta} \leq \frac{M_{2}^{-r} C_{1}^{r \delta}}{(2(p+2))^{r \delta}}\left(\|u\|_{2(p+2)}^{2(p+2)}+\|v\|_{2(p+2)}^{2(p+2)}\right)^{r \delta} .
$$

Since $L^{2(p+2)}(\Omega) \hookrightarrow L^{m+1}(\Omega), L^{2(p+2)}(\Omega) \hookrightarrow L^{r+1}(\Omega)$, we have

$$
\|u\|_{m+1}^{m+1} \leq C\|u\|_{2(p+2)}^{m+1}, \quad\|v\|_{r+1}^{r+1} \leq C\|v\|_{2(p+2)}^{r+1},
$$

thus

$$
\left(\|u\|_{2(p+2)}^{2(p+2)}+\|v\|_{2(p+2)}^{2(p+2)}\right)^{m \delta}\|u\|_{m+1}^{m+1} \leq C_{2}\left(\|u\|_{2(p+2)}^{2(p+2)}+\|v\|_{2(p+2)}^{2(p+2)}\right)^{m \delta+\frac{m+1}{2(p+2)}}
$$

and

$$
\left(\|u\|_{2(p+2)}^{2(p+2)}+\|v\|_{2(p+2)}^{2(p+2)}\right)^{r \delta}\|v\|_{r+1}^{r+1} \leq C_{3}\left(\|u\|_{2(p+2)}^{2(p+2)}+\|v\|_{2(p+2)}^{2(p+2)}\right)^{r \delta+\frac{r+1}{2(p+2)}}
$$

Using (3.2) and the following inequality:

$$
z^{v} \leq z+1 \leq\left(1+\frac{1}{a}\right)(z+a), \quad \forall z \geq 0,0<v \leq 1
$$

we obtain, for $t \geq 0$,

$$
\begin{aligned}
\left(\|u\|_{2(p+2)}^{2(p+2)}+\|v\|_{2(p+2)}^{2(p+2)}\right)^{m \delta+\frac{m+1}{2(p+2)}} & \leq d\left(\|u\|_{2(p+2)}^{2(p+2)}+\|v\|_{2(p+2)}^{2(p+2)}+H(0)\right) \\
& \leq d\left(\|u\|_{2(p+2)}^{2(p+2)}+\|v\|_{2(p+2)}^{2(p+2)}+H(t)\right)
\end{aligned}
$$

and

$$
\left(\|u\|_{2(p+2)}^{2(p+2)}+\|v\|_{2(p+2)}^{2(p+2)}\right)^{r \delta+\frac{r+1}{2(p+2)}} \leq d\left(\|u\|_{2(p+2)}^{2(p+2)}+\|v\|_{2(p+2)}^{2(p+2)}+H(t)\right),
$$

for $d=1+\frac{1}{H(0)}$. 
By (3.3)-(3.11) we have

$$
\begin{aligned}
\Theta^{\prime}(t) \geq & (1-\delta-M \varepsilon) H^{-\delta}(t) H^{\prime}(t)+\varepsilon\left(\frac{1}{j+1}+\frac{\gamma}{j+2}\right)\left(\left\|u_{t}\right\|_{j+2}^{j+2}+\left\|v_{t}\right\|_{j+2}^{j+2}\right) \\
& +\left(\gamma-K_{1}\right) \varepsilon H(t)+\varepsilon K_{2}\left(\|\nabla u\|_{\alpha}^{\alpha}+\|\nabla v\|_{\beta}^{\beta}\right)+\varepsilon K_{3}\left(\|\nabla u\|^{2}+\|\nabla v\|^{2}\right) \\
& +\varepsilon\left(\frac{\gamma}{2}-\lambda\right)(g \circ \nabla u+h \circ \nabla v)+\varepsilon\left(1+\frac{\gamma}{2}\right)\left(\left\|\nabla u_{t}\right\|^{2}+\left\|\nabla v_{t}\right\|^{2}\right) \\
& +\varepsilon\left(\frac{(2(p+2)-\gamma) C_{1}}{2(p+2)}-K_{1}\right)\left(\|u\|_{2(p+2)}^{2(p+2)}+\|v\|_{2(p+2)}^{2(p+2)}\right),
\end{aligned}
$$

where

$$
\begin{aligned}
& M=\frac{M_{1} m}{m+1}+\frac{M_{2} r}{r+1}, \\
& K_{1}=\frac{M_{1}^{-m} C_{1}^{m \delta} C_{2} d}{(m+1)(2(p+2))^{m \delta}}+\frac{M_{2}^{-r} C_{1}^{r \delta} C_{3} d}{(r+1)(2(p+2))^{r \delta}}, \\
& K_{2}=\min \left\{\frac{\gamma}{\alpha}-1, \frac{\gamma}{\beta}-1\right\}
\end{aligned}
$$

and

$$
K_{3}=\left(\frac{\gamma}{2}-1\right)-\left(\frac{\gamma}{2}-1+\frac{1}{4 \lambda}\right) \max \left(\int_{0}^{\infty} g(s) d s, \int_{0}^{\infty} h(s) d s\right) .
$$

Choose $M_{1}, M_{2}$ large enough such that

$$
\gamma-K_{1}>0, \quad \frac{(2(p+2)-\gamma) C_{1}}{2(p+2)}-K_{1}>0 .
$$

For fixed $M_{1}$ and $M_{2}$, we choose $\varepsilon>0$ small enough such that

$$
1-\delta-M \varepsilon \geq 0
$$

Then we can find positive constants $a_{1}$ and $a_{2}$ such that

$$
\begin{aligned}
\Theta^{\prime}(t) \geq & (1-\delta-M \varepsilon) H^{-\delta}(t) H^{\prime}(t)+\varepsilon\left(\frac{1}{j+1}+\frac{\gamma}{j+2}\right)\left(\left\|u_{t}\right\|_{j+2}^{j+2}+\left\|v_{t}\right\|_{j+2}^{j+2}\right) \\
& +\varepsilon K_{2}\left(\|\nabla u\|_{\alpha}^{\alpha}+\|\nabla v\|_{\beta}^{\beta}\right)+\varepsilon\left(1+\frac{\gamma}{2}\right)\left(\left\|\nabla u_{t}\right\|^{2}+\left\|\nabla v_{t}\right\|^{2}\right) \\
& +\varepsilon a_{1} H(t)+\varepsilon a_{2}\left(\|u\|_{2(p+2)}^{2(p+2)}+\|v\|_{2(p+2)}^{2(p+2)}\right) \geq 0 .
\end{aligned}
$$

Because of $H^{\prime}(t) \geq 0$, there exists constant $\Lambda>0$ such that

$$
\begin{aligned}
\Theta^{\prime}(t) \geq & \Lambda\left(H(t)+\left\|u_{t}\right\|_{j+2}^{j+2}+\left\|v_{t}\right\|_{j+2}^{j+2}+\|\nabla u\|_{\alpha}^{\alpha}+\|\nabla v\|_{\beta}^{\beta}\right. \\
& \left.+\left\|\nabla u_{t}\right\|^{2}+\left\|\nabla v_{t}\right\|^{2}+\|u\|_{2(p+2)}^{2(p+2)}+\|v\|_{2(p+2)}^{2(p+2)}\right) \geq 0 .
\end{aligned}
$$

On the other hand, we can choose $\varepsilon$ even smaller so that

$$
\Theta(0)=H^{1-\delta}(0)+\varepsilon \int_{\Omega}\left(u_{0} u_{1}+v_{0} v_{1}\right) d x>0 .
$$


Furthermore, we have

$$
\Theta(t) \geq \Theta(0), \quad t \geq 0 .
$$

Next we estimate $\Theta^{\frac{1}{1-\delta}}(t)$.

We have

$$
\begin{aligned}
& \Theta^{\frac{1}{1-\delta}}(t) \\
& \quad=\left(H^{1-\delta}(t)+\frac{\varepsilon}{j+1} \int_{\Omega}\left(\left|u_{t}\right|^{j} u_{t} u+\left|v_{t}\right|^{j} v_{t} v\right) d x-\varepsilon \int_{\Omega}\left(\Delta u u_{t}+\Delta v v_{t}\right) d x\right)^{\frac{1}{1-\delta}} \\
& \quad \leq C\left(H(t)+\left|\frac{\varepsilon}{j+1} \int_{\Omega}\left(\left|u_{t}\right|^{j} u_{t} u+\left|v_{t}\right|^{j} v_{t} v\right) d x-\varepsilon \int_{\Omega}\left(\Delta u u_{t}+\Delta v v_{t}\right) d x\right|^{\frac{1}{1-\delta}}\right) .
\end{aligned}
$$

By Hölder's inequality, the Sobolev embedding theorem, and Young's inequality, we get

$$
\begin{aligned}
& \left|\frac{\varepsilon}{j+1} \int_{\Omega}\left(\left|u_{t}\right|^{j} u_{t} u+\left|v_{t}\right|^{j} v_{t} v\right) d x-\varepsilon \int_{\Omega}\left(\Delta u u_{t}+\Delta v v_{t}\right) d x\right|^{\frac{1}{1-\delta}} \\
& \leq C\left(\|u\| \frac{\frac{1}{1-\delta}}{1-u_{t}}\left\|^{\frac{j+1}{1-\delta}}+\right\| v\left\|^{\frac{1}{1-\delta}}\right\| v_{t}\left\|^{\frac{j+1}{1-\delta}}+\right\| \nabla u\left\|^{\frac{1}{1-\delta}}\right\| \nabla u_{t}\left\|^{\frac{1}{1-\delta}}+\right\| \nabla v\left\|\frac{1}{1-\delta}\right\| \nabla v_{t} \|^{\frac{1}{1-\delta}}\right) \\
& \leq C\left(\|u\|_{2(p+2)}^{\frac{s}{1-\delta}}+\left\|u_{t}\right\|_{j+2}^{\frac{(j+1) \tau}{1-\delta}}+\|v\|_{2(p+2)}^{\frac{s}{1-\delta}}+\left\|v_{t}\right\|_{j+2}^{\frac{(j+1) \tau}{1-\delta}}+\|\nabla u\|_{\alpha}^{\frac{\lambda}{1-\delta}}\right. \\
& \left.\quad+\left\|\nabla u_{t}\right\|^{\frac{\mu}{1-\delta}}+\|\nabla v\|_{\beta}^{\frac{\lambda}{1-\delta}}+\left\|\nabla v_{t}\right\|^{\frac{\mu}{1-\delta}}\right),
\end{aligned}
$$

where

$$
\begin{aligned}
& \frac{1}{s}+\frac{1}{\tau}=1, \\
& \frac{1}{\lambda}+\frac{1}{\mu}=1 .
\end{aligned}
$$

Set

$$
\frac{(j+1) \tau}{1-\delta}=j+2, \quad \mu=2(1-\delta)
$$

thus

$$
\frac{s}{1-\delta}=\frac{j+2}{1-(j+2) \delta}, \quad \frac{\lambda}{1-\delta}=\frac{2}{1-2 \delta} .
$$

Furthermore, we have

$$
\begin{aligned}
& \|u\|_{2(p+2)}^{\frac{j+2}{1-(j+2) \delta}} \leq d\left(\|u\|_{2(p+2)}^{2(p+2)}+H(t)\right), \\
& \|v\|_{2(p+2)}^{\frac{j+2}{1-(j+2) \delta}} \leq d\left(\|v\|_{2(p+2)}^{2(p+2)}+H(t)\right), \\
& \|\nabla u\|_{\alpha}^{\frac{2}{1-2 \delta}} \leq d\left(\|\nabla u\|_{\alpha}^{\alpha}+H(t)\right), \\
& \|\nabla v\|_{\beta}^{\frac{2}{1-2 \delta}} \leq d\left(\|\nabla v\|_{\beta}^{\beta}+H(t)\right)
\end{aligned}
$$


and

$$
\begin{aligned}
& \left|\frac{\varepsilon}{j+1} \int_{\Omega}\left(\left|u_{t}\right|^{j} u_{t} u+\left|v_{t}\right|^{j} v_{t} v\right) d x-\varepsilon \int_{\Omega}\left(\Delta u u_{t}+\Delta v v_{t}\right) d x\right|^{\frac{1}{1-\delta}} \\
& \leq C\left(H(t)+\left\|u_{t}\right\|_{j+2}^{j+2}+\left\|v_{t}\right\|_{j+2}^{j+2}+\|\nabla u\|_{\alpha}^{\alpha}+\|\nabla v\|_{\beta}^{\beta}+\left\|\nabla u_{t}\right\|^{2}+\left\|\nabla v_{t}\right\|^{2}\right. \\
& \left.\quad+\|u\|_{2(p+2)}^{2(p+2)}+\|v\|_{2(p+2)}^{2(p+2)}\right) .
\end{aligned}
$$

Thus we obtain

$$
\begin{aligned}
\Theta^{\frac{1}{1-\delta}}(t) \leq & C\left(H(t)+\left\|u_{t}\right\|_{j+2}^{j+2}+\left\|v_{t}\right\|_{j+2}^{j+2}+\|\nabla u\|_{\alpha}^{\alpha}+\|\nabla v\|_{\beta}^{\beta}\right. \\
& \left.+\left\|\nabla u_{t}\right\|^{2}+\left\|\nabla v_{t}\right\|^{2}+\|u\|_{2(p+2)}^{2(p+2)}+\|v\|_{2(p+2)}^{2(p+2)}\right) .
\end{aligned}
$$

A combination of (3.12) and (3.16) yields

$$
\Theta^{\prime}(t) \geq \zeta \Theta \frac{1}{1-\delta}(t)
$$

where $\zeta$ is some positive constant. A simple integration gives

$$
\Theta^{\delta /(1-\delta)} \geq \frac{1}{\Theta^{-\delta /(1-\delta)}(0)-\frac{\zeta \delta t}{1-\delta}} .
$$

Thus $H(t)$ blows up in a finite time, and the estimation of the blow-up time $T^{*}$ obeys

$$
T^{*} \leq \frac{1-\delta}{\zeta \delta \Theta^{\delta /(1-\delta)}(0)}
$$

This completes the proof of Theorem 3.1.

\section{Competing interests}

The authors declare that they have no competing interests.

\section{Authors' contributions}

The authors declare that the study was realized in collaboration with the same responsibility. All authors read and approved the final manuscript.

\section{Acknowledgements}

The authors would like to express their gratitude to the anonymous referees for helpful and fruitful comments, and very careful reading on this paper. This research was partially supported by National Natural Science Foundation of China (61374089), Natural Science Foundation of Shanxi Province (2014011005-2), Shanxi Scholarship Council of China (2013-013), and Shanxi International Science and Technology Cooperation Projects (2014081026).

Received: 29 October 2014 Accepted: 18 November 2014 Published online: 28 November 2014

\section{References}

1. Dafermos, CM: Asymptotic stability in viscoelasticity. Arch. Ration. Mech. Anal. 37, 297-308 (1970)

2. Hrusa, WJ: Global existence and asymptotic stability for a semilinear Volterra equation with large initial data. SIAM J. Math. Anal. 16(1), 110-134 (1985)

3. Muñoz Rivera, JE: Asymptotic behavior in linear viscoelasticity. Q. Appl. Math. 52(4), 628-648 (1994)

4. Messaoudi, SA: Blow up and global existence in a nonlinear viscoelastic wave equation. Math. Nachr. 260, 58-66 (2003)

5. Messaoudi, SA: Blow up of positive-initial-energy solutions of a nonlinear viscoelastic hyperbolic equation. J. Math. Anal. Appl. 320, 902-915 (2006)

6. Cavalcanti, MM, Oquendo, HP: Frictional versus viscoelastic damping in a semilinear wave equation. SIAM J. Control Optim. 42(4), 1310-1324 (2003) 
7. Cavalcanti, MM, Domingos Cavalcanti, VN, Lasiecka, I, Nascimento, FAF: Intrinsic decay rate estimates for the wave equation with competing viscoelastic and frictional dissipative effects. Discrete Contin. Dyn. Syst., Ser. B 19(7), 1987-2012 (2014)

8. Muñoz Rivera, JE, Naso, MG: On the decay of the energy for systems with memory and indefinite dissipation. Asymptot. Anal. 49(3-4), 189-204 (2006)

9. Wang, J, Wang, JM: Spectral analysis and exponential stability of one-dimensional wave equation with viscoelastic damping. J. Math. Anal. Appl. 410(1), 499-512 (2014)

10. Zhao, DX, Wang, JM: Spectral analysis and stabilization of a coupled wave-ODE system. J. Syst. Sci. Complex. 27(3), 463-475 (2014)

11. Cavalcanti, MM, Domingos Cavalcanti, VN, Ferreira, J: Existence and uniform decay for nonlinear viscoelastic equation with strong damping. Math. Methods Appl. Sci. 24, 1043-1053 (2001)

12. Messaoudi, SA, Tatar, NE: Global existence and uniform stability of solutions for a quasilinear viscoelastic problem. Math. Methods Appl. Sci. 30, 665-680 (2007)

13. Messaoudi, SA, Tatar, NE: Exponential and polynomial decay for a quasilinear viscoelastic equation. Nonlinear Anal. TMA 68, 785-793 (2007)

14. Pata, V: Exponential stability in linear viscoelasticity. Q. Appl. Math. LXIV(3), 499-513 (2006)

15. Lasiecka, I, Messaoudi, SA, Mustafa, M: Note on intrinsic decay rates for abstract wave equations with memory. J. Math. Phys. 54(3), 031504 (2013)

16. Han, X, Wang, M: Global existence and blow-up of solutions for a system of nonlinear viscoelastic wave equations with damping and source. Nonlinear Anal. TMA 71, 5427-5450 (2009)

17. Messaoudi, SA, Said-Houari, B: Global nonexistence of positive initial-energy solutions of a system of nonlinear viscoelastic wave equations with damping and source terms. J. Math. Anal. Appl. 365, 277-287 (2010)

18. Said-Houari, B, Messaoudi, SA, Guesmia, A: General decay of solutions of a nonlinear system of viscoelastic wave equations. Nonlinear Differ. Equ. Appl. 18, 659-684 (2011)

19. Liu, W: Uniform decay of solutions for a quasilinear system of viscoelastic equations. Nonlinear Anal. TMA 71, 2257-2267 (2009)

doi:10.1186/s13661-014-0250-z

Cite this article as: Hao et al.: Global nonexistence of solutions for nonlinear coupled viscoelastic wave equations with damping and source terms. Boundary Value Problems 2014 2014:250.

\section{Submit your manuscript to a SpringerOpen ${ }^{\ominus}$ journal and benefit from:}

- Convenient online submission

Rigorous peer review

- Immediate publication on acceptance

Open access: articles freely available online

- High visibility within the field

- Retaining the copyright to your article 